\title{
Effect of Orthotopic Liver Transplantation on the Health-Related Quality of Life in Indian Patients with End-Stage Liver Disease: A Prospective Study
}

Vinayak Nikam ( $\nabla$ vinayaknikam7183@gmail.com )

Sir Ganga Ram Hospital https://orcid.org/0000-0002-6648-6811

Vasudevan Ramaswamy

Sir Ganga Ram Hospital

Abhideep Chaudhary

Sir Ganga Ram Hospital

Suresh Singhvi

Sir Ganga Ram Hospital

Research article

Keywords: Health-Related Quality of Life, Model for End-Stage Liver Disease, Child-Turcotte-Pugh Score, Orthotopic Liver transplantation, SF-36 questionnaire

Posted Date: September 17th, 2020

DOl: https://doi.org/10.21203/rs.3.rs-71536/v1

License: (c) (i) This work is licensed under a Creative Commons Attribution 4.0 International License. Read Full License 


\section{Abstract \\ BACKGROUND}

Health-related quality of life (HRQOL) is an important measure of the effects of end stage liver disease in affected patients that helps to improve well-being after Liver transplantation (LT). One study has been performed to access HRQoL pre and post liver transplant (LT) patients in India. Our study was aimed to determine HRQoL in pre and post orthotopic liver transplant (OLT) with correlation between severity of liver disease by Model for End-Stage Liver Disease (MELD)/Child-Turcotte-Pugh (CTP) score and Diabetes Mellitus (DM) status with HRQoL post OLT.

\section{METHODS}

Study was designed as a single center, observational, prospective and longitudinal study. HRQoL was evaluated by SF-36 questionnaire in sixty (50 male and 10 female with average age of 45 years) patients with end stage liver disease; having HCV related cirrhosis with alcoholic and cryptogenic liver disease forming the second and third largest groups respectively. HRQoL was evaluated pre and post OLT also correlation of MELD/CTP scores and diabetes status with post-OLT HRQoL was determined. Patients were followed up for a period of 3 months after OLT.

\section{RESULTS}

Post OLT patients had higher scores in comparison to pre OLT. There was a significant difference in all eight domains of the SF-36 scores $(p<0.001)$ post OLT. Negative correlation was observed between MELD/CTP scores and HRQoL post OLT with significance in the domain of vitality and mental component summary in MELD and physical functioning in CTP $(p<0.05)$. Diabetes did not exert any significant effect on HRQoL.

\section{CONCLUSIONS}

OLT have an improved HRQoL when compared to their pre transplant status. MELD/CTP scores were not predictive of HRQoL post OLT, while DM status did not exhibit any effect on HRQoL after OLT.

\section{Introduction}

End-stage liver disease is a progressive illness with liver transplantation (LT) as an established form of treatment ${ }^{1}$. In India with a population of 1.37 billion till March 2019, the end-stage liver disease is becoming increasingly prevalent with an estimated annual requirement of LT between 30,000 and 32,000. 
Currently, only about 2200 to 2500 liver transplants operations are being performed due to limited cadaveric donor pool ${ }^{2,3}$ and the approved number of government recognized centers ${ }^{4}$.

Liver transplantation is the gold standard therapeutic option for end-stage liver disease ${ }^{5}$. In the last few decades, there have been progressive improvements in graft and patient survival ${ }^{6}$. The1, 5- and 10-years survival has been reported to be $92 \%, 83 \%$, and $79 \%$, respectively ${ }^{7}$. Etiological categorization of end-stage liver disease revealed the hepatitis $C$ virus ( $\mathrm{HCV}$ ), hepatitis B virus (HBV), ethanol-related, and cryptogenic cirrhosis as a major cause of liver damage ${ }^{8}$. Patients with the end-stage Liver disease have a negative impact on daily health-related quality of life (HRQoL). As life expectancy after liver transplantation continuously improving, there has been a transit from the medical management of LT recipients to a more long-term approach in form of HRQoL, which includes not only the physical well-being of recipients but also their social and emotional well-being.

The quality of life of recipients evaluated by several studies and has determined important elements such as medical complications, physical function, social function, fatigue, employment status, emotional function, and sexual dysfunction ${ }^{9}$. These studies have shown that the quality of life improves after LT; this is demonstrated by improvements in a recipient's functional capacity, perception of health condition, and self-esteem and these parameters continue to revamp over time. Short form-36(SF-36) is a validated questionnaire derived from a larger set of questions and provides a composite scale of mental and physical health for patients. Studies published outside India reported positive outcomes in HRQoL after $\mathrm{LT}^{10,11,12}$. In Indian scenario two studies have been reported with one determining HRQoL using SF-36 in liver cirrhosis patients compared with the same age and gender healthy controls, the severity of liver disease in this study was assessed by MELD and CTP scoring ${ }^{13}$ while in another study Bidare et $\mathrm{al}^{14}$ assed HRQoL using SF-36 in pre and post LT; hence this study will provide more insight on HRQoL in pre and post LT Indian patient's, Also it will give information on the correlation between the severity of liver disease (MELD and CTP) and Diabetes mellitus (DM).

\section{Material And Methods}

The study was designed as a prospective, observational, and single-center study. Sixty end-stage liver disease patients evaluated for LT at Sir Ganga Ram Hospital, New Delhi between June 2013 to March 2015 in the department of Surgical Gastroenterology and Liver Transplantation were enrolled in the study. This study is approved by the Institutional ethics committee board (EC/08/13/586). Informed and written consent for participation was obtained from all the recipients and they all completed the validated SF-36 questionnaire by interview or E-mail pre-LT as well as Post-LT follow-up at 3 months. The study was performed under a license approval certificate (License Number- QM029912, Optuminsight Life Sciences, Inc) for the use of the SF-36 software. The results from questionnaires completed by interview versus mail were equivalent. In this study chronic end-stage liver disease patients were included like HCV-related cirrhosis, alcoholic liver cirrhosis, HBV-related cirrhosis, Autoimmune, Budd Chiari Syndrome, and nonalcoholic fatty liver (NASH). The other inclusion criteria were age $\geq 18$ years, a fully-conscious, 
ambulatory, liver transplant recipient. Patients with acute liver failure, multiple-organ transplant listed, and patients not willing to participate or complete the SF-36 questionnaire were excluded.

\section{Post-transplant Follow-up protocol}

Our institute developed its own post - LT follow-up protocol model to assess the physical and psychosocial recovery of our recipients. At the time of discharge, recipients and their family members were educated about healthy lifestyle and general daily life precautions. It was also suggested that patients should visit transplant physicians or surgeons monthly in the first 6 months, every 3 monthly in the second 6 months, and 6 monthly beyond 1 year. Recipients were instructed to follow up with our transplant physicians in the out-patient clinic or by mail or telephone if they had any health-related problems.

Regularly, our institute staff also doing telephonic communication with the recipient to know general health. Patients were further evaluated with clinical and laboratory investigation stools, and intervention or even readmission, in case any abnormalities were identified. Furthermore, an annual meeting was held to update our recipients with information related to medication and daily care, and maintaining optimum HRQoL was emboldened at the annual meeting.

\section{SF-36- Tool to evaluate HRQoL}

The SF-36 questionnaire was used to evaluate HRQoL consisting 36 questions (items) measuring physical and mental health status with eight health parameters: physical functioning (PF), role limitations due to physical problems: role physical (PR), body pain (BP), general health perceptions (GH), vitality: energy/fatigue (VT), social function (SF), role limitations due to emotional problems: role emotional (ER), and general mental health: psychological distress/wellbeing (MH). The SF-36 questionnaire was given to the recipient's pre and post-liver transplantation by interview or mail-in language he/she understands (Hindi and English version). Responses to each of the SF-36 items were scored and summed according to a standardized scoring protocol and expressed as a score on a 0-100 scale for each of the eight health concepts. Higher scores represent better self-perceived health. The maximum of 100 on these bipolar scales, therefore, indicated not just the absence of disability, but the presence of a positive state of health. Two-component summary measures have been calculated from eight health parameters: The Physical Component Score (PCS) and Mental Component Score (MCS). The summary scores were aggregated measures of the physical health and mental health dimensions underlying the SF-36 questionnaire. The severity of liver disease was assessed before transplant with MELD and CTP scores, with the diabetes status of the patients. The correlation between the severity of disease (MELD, CTP) and DM status with HRQoL at 3 months post-transplant was also evaluated.

\section{Statistical analysis}

Statistical data were analyzed by using Statistical Package for the Social Sciences (SPSS), version 17. Data values were calculated using software provided by the SF36 health questionnaire and reported as 
mean \pm SD. The Categorical numbers were expressed as percentages and frequencies. Nominal categorical variables between the groups were compared using a nonparametric sign test, while the Paired t-test was used to compare the normally distributed continuous variables pre-operative and postoperative 3 months. Spearman correlation analysis was used to analyze the relationships between HRQoL and pre-transplantation severity of liver disease and DM status, a p-value of $<0.05$ was considered statistically significant.

\section{Results}

\section{Recipient Characteristics}

The demographic characteristics of the study population are listed in Table 1. Most recipient's average age was 45 years at the time of transplantation and male (83.3\%) to female $(16.7 \%)$ ratio was $5: 1$.

Classification by primary liver disease at the time of referral for transplantation showed that the largest group had HCV related cirrhosis with alcoholic and cryptogenic liver disease forming the second and third largest groups respectively. Among the participants, 13.3\% had Hepatocellular carcinoma (HCC), 26.7\% had Spontaneous bacterial peritonitis (SBP), 36.7\% experienced Hepato-renal syndrome (HRS) and18.3\% had Diabetes mellitus (DM), while $16.7 \%$ reported that they experienced early postoperative sepsis. All recipients underwent living donor LT, all of whom were first or second-degree relatives of their donors. 
Table 1

Demographics and clinical variables of the patients

\begin{tabular}{|ll|}
\hline Variable & $\mathbf{n}=60$ \\
\hline Age (Mean \pm SD) & $45 \pm 10$ \\
\hline Gender & \\
\hline Male & $50(83.3 \%)$ \\
\hline Female & $10(16.7 \%)$ \\
\hline Child-Turcotte-Pugh Score & \\
\hline A & $2(3.3 \%)$ \\
\hline B & $9(15 \%)$ \\
\hline C & $49(81.7 \%)$ \\
\hline Model for End-stage Liver Disease & \\
\hline$<20$ & $13(21.7 \%)$ \\
\hline$\geq 20$ & $47(78.3 \%)$ \\
\hline Etiology & \\
\hline Hepatitis C virus & $25(41.7 \%)$ \\
\hline Alcoholic & $15(25.0 \%)$ \\
\hline Cryptogenic & $9(15 \%)$ \\
\hline Hepatitis B virus & $7(11.7 \%)$ \\
\hline Auto Immune Liver Disease & $2(3.3 \%)$ \\
\hline Budd-Chiari Syndrome & $1(1.7 \%)$ \\
\hline Non-alcoholic Steatohepatitis & $1(1.7 \%)$ \\
\hline Hepatocellular carcinoma & $52(86.7 \%)$ \\
\hline Spontaneous bacterial peritonitis & $16(26.7 \%)$ \\
\hline Hepato-renal syndrome & $22(36.7 \%)$ \\
\hline Diabetes mellitus & $11(18.3 \%)$ \\
\hline Post-operative Sepsis & \\
\hline
\end{tabular}

HRQoL scores Pre and Post-transplant. 
The HRQoL scores of pre and post-transplant are mentioned in Table 2 and Fig. 1 There was a great difference $(p<0.001)$ in scores of eight domains of the SF-36. Compared to the pre-transplantation, significant improvements were seen in the post-liver transplant values in terms of PF, PR, BP, GH, VT, SF, $\mathrm{RE}$, and $\mathrm{MH}$. Overall there was a significant change in Mental Component Summary and Physical Component Summary in post-transplant as compared to the pre-transplant.

Table 2

Pre and post-transplant HRQoL scores (mean $\pm \mathrm{SD}$ ).

\begin{tabular}{|llll|}
\hline SF-36 & Pre-transplant & Post-transplant & p Value \\
\hline Physical Functioning (PF) & $29.83 \pm 21.59$ & $81.00 \pm 17.09$ & $\mathrm{p}<0.001$ \\
\hline Physical Role (PR) & $12.08 \pm 30.36$ & $80.00 \pm 33.45$ & $\mathrm{p}<0.001$ \\
\hline Bodily Pain (BP) & $41.25 \pm 17.72$ & $83.12 \pm 18.80$ & $\mathrm{p}<0.001$ \\
\hline General Health (GH) & $13.37 \pm 15.01$ & $75.28 \pm 17.53$ & $\mathrm{p}<0.001$ \\
\hline Vitality (VT) & $27.92 \pm 17.18$ & $76.25 \pm 12.54$ & $\mathrm{p}<0.001$ \\
\hline Social Functioning (SF) & $32.71 \pm 17.99$ & $76.85 \pm 14.87$ & $\mathrm{p}<0.001$ \\
\hline Emotional Role (ER) & $15.55 \pm 34.43$ & $86.67 \pm 28.92$ & $\mathrm{p}<0.001$ \\
\hline Mental Health (MH) & $36.13 \pm 19.19$ & $79.67 \pm 11.41$ & $\mathrm{p}<0.001$ \\
\hline Physical Component Summary (PCS) & $29.08 \pm 7.29$ & $50.00 \pm 7.44$ & $\mathrm{p}<0.001$ \\
\hline Mental Component & $30.96 \pm 9.09$ & $52.89 \pm 6.09$ & $\mathrm{p}<0.001$ \\
\hline Summary (MCS) & & & \\
\hline
\end{tabular}

Correlations between pre-transplant severity of liver disease, diabetes status, and HRQoL post-transplant.

The results of the Spearman correlation test for determination of correlations between pre-transplant severity of liver disease (MELD/CTP)/DM status and HRQoL (post-transplant) assessed with SF 36 are shown in Table 3.There was a negative correlation between overall SF-36 scores and MELD/CTP scores. In MELD negative correlation was found in VT $(r=-0.32, p<0.05)$ and MCS $(r=-30, p<0.05)$, while in CTP negative correlation was observed in $\mathrm{PF}(r=-0.30, p<0.05)$, which were found to be significant. No significant correlation was observed between the presence of diabetes mellitus and HRQoL after OLT. 
Table 3

The $\mathrm{p}$ values (correlation coefficient) between SF-36 post OLT with MELD / CTP and DM status.

\begin{tabular}{|c|c|c|c|c|c|c|}
\hline \multirow[t]{2}{*}{ SF-36 } & \multicolumn{2}{|c|}{ MELD } & \multicolumn{2}{|l|}{ CTP } & \multicolumn{2}{|l|}{ DM } \\
\hline & $r$ & p & $r$ & $\mathbf{p}$ & $r$ & p \\
\hline PF & -0.25 & 0.06 & -0.30 & $0.02 *$ & -0.089 & 0.50 \\
\hline PR & -0.24 & 0.07 & -0.08 & 0.54 & -0.092 & 0.48 \\
\hline BP & -0.21 & 0.11 & -0.19 & 0.14 & -0.19 & 0.15 \\
\hline $\mathrm{GH}$ & -0.18 & 0.17 & -0.01 & 0.93 & -0.02 & 0.90 \\
\hline VT & -0.32 & $0.01 *$ & -0.14 & 0.29 & 0.02 & 0.92 \\
\hline SF & -0.16 & 0.20 & -0.15 & 0.24 & 0.01 & 0.97 \\
\hline ER & -0.25 & 0.05 & -0.19 & 0.14 & -0.059 & 0.66 \\
\hline $\mathrm{MH}$ & -0.25 & 0.05 & -0.08 & 0.52 & -0.013 & 0.92 \\
\hline PCS & -0.25 & 0.05 & -0.16 & 0.23 & -0.16 & 0.23 \\
\hline MCS & -0.30 & $0.02^{*}$ & -0.15 & 0.25 & -0.013 & 0.92 \\
\hline \multicolumn{7}{|c|}{$\begin{array}{l}\text { Abbreviations: PF, physical functioning; PR, role physical; BP, bodily pain; GH, general health; VT, } \\
\text { vitality; SF, social functioning; ER, role emotional; MH, mental health; PCS, physical component score; } \\
\text { MCS, mental component score, MELD: Model for End-stage Liver Disease, CTP: Child-Turcotte-Pugh, } \\
\text { DM: Diabetes mellitus. }\end{array}$} \\
\hline
\end{tabular}

\section{Discussion}

The current study analyzed pre and post-liver transplant recipients HRQoL by using the SF-36 questionnaire, the pre-transplant patients were found to have low HRQoL scores indicating the altered quality of life. End-stage liver disease has a negative effect on all aspects of a patient's well-being and HRQoL ${ }^{15}$. Hence improving the patient's quality of life after liver transplantation takes on increasing importance ${ }^{16}$. The survival after LT is optimally increased hence healthcare providers and patients are more concerned about post-transplant HRQoL.

HRQoL focuses on patient care, with the improvement in patient and recipient survival after $\mathrm{LT}^{17}$. In the present study, there was a significant improvement in all the domains of SF-36 related to HRQoL after 3 months of OLT. Telles-Correia et al found a significant increase in mental and physical component scores at 1 month after $\mathrm{LT}^{18}$. Radcliffe et al. ${ }^{19}$ showed that at 3 months post-OLT, there was a statistically great improvement in HRQoL scores in all SF-36 domains except bodily pain. De Bona et al ${ }^{20}$ reported an increase in the score for the first 6 months with a decrease in 13 to 24 months and improvement between 
3 and 5 years. A study by Younoussi et al. ${ }^{21}$ stated that an increase in all SF-36 HRQoL parameters within the first 6 months post-OLT. Likewise, Krasnoff et al. ${ }^{22}$ studied post-LT HRQoL scores at 2, 6, 12, and 24 months after OLT, there was an enormous increase in SF-36 physical function, role function, bodily pain and general health domain scores at both 2 and 6 months. Thus significant improvement in HRQoL was observed in Indian patients after OLT from our study indicating positive outcomes in both mental and physical health status.

\section{The severity of liver disease (CTP and MELD Score) and HRQoL}

In our study MELD/CTP showed a negative correlation in all the SF-36 domains with a significant negative correlation in vitality (VT) and mental component score (MCS) in MELD and Physical functioning (PF) in CTP score. One might expect the liver disease severity negatively correlate with HRQoL scores. Saab et al. ${ }^{23}$ attempted to correlate the severity of liver disease by the MELD and CTP scoring with pre-transplantation HRQoL scores and found that the MELD score was not predictive of HRQoL scores, with the SF-36 used as an assessment instrument. The presence of hepatic encephalopathy and/or ascites in ESLD is significantly associated with unfavorable quality of life. CTP score comprises of hepatic encephalopathy and ascites, due to this reason CTP more appropriately correlate with QOL. Estraviz et al ${ }^{25}$ found that the CTP score could be correlated with pre-transplantation HRQoL parameters at 6 months after transplant. Castaldo et al ${ }^{26}$ reported that increased MELD score before grafting was associated with improved physical HRQoL in the first 12 months after LT and did not affect mental HRQoL. Rodrigue et al failed to show a correlation between CTP score and HRQoL, but they found a good correlation between MELD Score and HRQoL.

Kotarska et $\mathrm{al}^{27}$ reported that liver severity analyzed with CTP and MELD scores before LT had a minimal effect on HRQoL after LT and exerts an insignificant effect on patients evaluated 1 year after LT. Few studies showed that there is no correlation between HRQoL and severity of the liver disease like Ortega et $\mathrm{al}^{24}$ reported that MELD and CTP scores did not correlate with HRQoL either before or after transplantation.

\section{Diabetes mellitus and HRQoL}

In this study, the presence of diabetes mellitus did not have any significant correlation with HRQoL after OLT. Kotarska et $\mathrm{al}^{27}$ experienced that the recipients with DM seemed to have an unsatisfactory quality of life early after surgery.

\section{Limitations Of The Study}

Our study has a few important limitations. First, relatively few patients were enrolled in the study. Thus, it would be appropriate to extend it to include more number of patients. Second, the study had short term follow up and HRQoL assed once only after the first 3 months post $L T$, hence it is very difficult to assess long term HRQoL. Third, in this study pre LT patients were compared with post LT patients, however, in 
other studies post LT recipients were compared with the healthy general population. Lastly, there are many studies in which employment, income, sexual and reproductive parameters analyzed after transplant, but the present study didn't assed these.

In conclusion, there was a significant increase in HRQoL at first quarter post-LT with no prediction between the severity of disease and diabetes status in Indian patients. However, HRQoL and correlation between severities of liver disease should be evaluated for a longer duration of time on larger Indian liver transplant population

\section{Abbreviations}

DDLT- Deceased donor liver transplant

LDLT- Living donor liver transplant

DDA- Duct-to-duct biliary anastomosis

ESLD-End stage liver disease

UW -University of Wisconsin solution

CUSA- cavitronic ultrasonic surgical aspirator

IOC- Intra-operative cholangiogram

HV-Hepatic vein

EGD- Early graft dysfunction

DCD- Donation after circulatory

MELD-Model for end stage liver disease

MRE-Magnetic resonance elastography

GRWR-Graft to recipient weight ratio

CIT- Cold Ischemia Time

WIT-Warm Ischemia Time

LAl- Liver Attenuation Index

\section{Declarations}


1. Institutional Ethics Committee, Sir Ganga Ram Hospital, New Delhi, India (EC/08/13/586). 2. All

Recipients gave informed written consent for participation in study.

\section{Consent for publication}

Not Applicable

\section{Availability of data and material}

The data sets used and/or analyzed during the current study are available from the corresponding author on reasonable request.

\section{Competing interests}

The authors declare that they have no competing interests.

\section{Funding}

No grant support or any kind of assistance from other institute

\section{Authors' contributions}

A) Substantial contributions to the conception or design of the work; or the acquisition, analysis, or interpretation of data for the work -VN, VR, AC, SS

B) Drafting the work or revising it critically for important intellectual content- VN, VR, AC, SS

C) Final approval of the version to be published- VN, VR, AC, SS

D) Agreement to be accountable for all aspects of the work in ensuring that questions related to the accuracy or integrity of any part of the work are appropriately investigated and resolved- VN, VR, AC, SS

\section{Acknowledgements}

The authors would like to thank Anusha Nikam and Jay Hire for the language editing help and technical support.

\section{References}

1. Jamie Potosek M, Curry M, Buss, Eva Chittenden. Integration of Palliative Care in End-Stage Liver Disease and Liver Transplantation. Journal of Palliative Medicine. 2014;17:11.

2. Narasimhan G, Kota V, Rela M. Liver transplantation in India. Liver Transpl. 2016;22:1019-24.

3. Viniyendra Pamecha DP, Borle S, Kumar. Kishore Gurumoorthy Subramanya Bharathy, Piyush Kumar Sinha, Shridhar Vasantrao Sasturkar et al. Deceased donor liver transplant: Experience from a public sector hospital in India. Indian J Gastroenterol2018; 37(1):18-24. 
4. Nagral S, Nanavati A, Nagral A. Liver Transplantation in India: At the Crossroads. Journal of Clinical Experimental Hepatology. 2015;5:329-40.

5. Neuberger J. What is the real gain after liver transplantation? Liver Transpl. 2009;15(11):1-5.

6. Roberts MS, Angus DC, Bryce CL, Weissfeld L, Valenta Z. Survival after liver transplantation in the United States: A disease-specific analysis of the UNOS database. Liver Transpl. 2004;10:886-97.

7. Soin AS, Thiagarajan S. Liver transplant scene in India. MAMC Journal of Medical Sciences. 2016;2:6-11.

8. Nayak NC, Jain D, Vasdev N, Gulwani H, Saigal S, et al. Etiologic types of end-stage chronic liver disease in adults: analysis of prevalence and their temporal changes from a study on native liver explants. Eur J Gastroenterol Hepatol. 2012;24(10):1199-208.

9. Burra P. Sexual dysfunction after liver transplantation. Liver Transpl. 2009;15(2):50-6.

10. Desai R, Jamieson NV, Gimson AE, Watson CJ, Gibbs P, et al. Quality of Life up to 30 years Following Liver Transplantation. Liver Transpl. 2008;14:1473-9.

11. Wang G-S, Yang Y, Li H, Jiang N, Fu B-S, et al. Health-related quality of life after liver transplantation: the experience from a single Chinese center. HepatobiliaryPancreat Dis Int. 2012;11:262-6.

12. Chen P-X, Wang Lu-NanYWen-Tao. Health-related quality of life of 256 recipients after liver transplantation. World J Gastroenterol. 2012;18(36):5114-21.

13. Janani K, Varghese J, Jain M, Harika K, Srinivasan V, et al. HRQOL using SF36 (generic specific) in liver cirrhosis. Indian Society of Gastroenterology. 2017;36(4):313-7.

14. Asha Bidare K, Raja S, Asthana JR, Lochan, Mathew Jacob. Effect of liver transplantation on the health-related quality of life (HRQoL) of a patient with end-stage liver failure. Indian Journal of Transplantation. 2014;8:126-38.

15. Younossi Z, Guyatt G. Quality of life assessments in chronic liver disease. Am J Gastroenterol. 1998;93:1037-41.

16. Belle SH, Porayko MK, Hoofnagle JH, Lake JR, Zetterman RK. Changes in quality of life after liver transplantation among adults. Liver Transpl Surg. 1997;3:93-104.

17. Bownik H, Saab S. Health-related quality of life after liver transplantation for adult recipients. Liver Transpl. 2009;15:42-9.

18. Telles-Correia D, Barbosa A, Mega I, Mateus E, Monteiro E. When does quality of life improve after liver transplantation? A longitudinal prospective study. Transpl Proc. 2009;41:904-5.

19. Ratcliffe J, Longworth L, Young T, Bryan S, Burroughs A, et al. Cost-effectiveness of liver transplantation team assess health-related quality of life pre- and post-liver transplantation: a prospective multicenter study. Liver Transpl. 2002;8:262-70.

20. De Bona M, Ponton P, Ermani M, Lemmolo RM, Feltrin A, et al. The impact of liver disease and medical complications on quality of life and psychological distress before and after liver transplantation. J Hepatol. 2000;33:609-15. 
21. Younoussi ZM, McCormick M, Price LL, Boparai N, Farquhar L, et al. Impact of liver transplantation on health-related quality of life. Liver Transpl. 2000;6:779-83.

22. Krasnoff JB, Vintro AQ, Ascher NL, Bass NM, Dodd MJ, et al. Objective measures of health-related quality of life over 24 months post-liver transplantation. Clin Transplant. 2005;19:1-9.

23. Saab S, Ibrahim AB, Shpaner A, Younossi ZM, Lee C, et al. MELD fails to measure the quality of life in liver transplant candidates. Liver Transpl. 2005;11:218-23.

24. Ortega T, Deulofeu R, Salamero P, Casanovas T, Rimola A, et al. Impact of Health-Related Quality of Life in Catalonia Liver Transplant Patients. Transplantation Proceedings 2009; 41:2187-2188.

25. Estraviz B, Quintana JM, Valdivieso A, Bilbao A, Padierna A, et al. Factor influencing change in healthrelated quality of life after liver transplantation. Clin Transplant. 2007;21:481-90.

26. Castaldo ET, Feurer ID, Russell RT, Pinson CW. Correlation of health-related quality of life after liver transplant with the Model for End-Stage Liver Disease score. Arch Surg. 2009;144(2):167-72.

27. Kotarska K, Raszeja-Wyszomirska J, Wunsch E, Chmurowiczd T, Kempinska-Podhorodecka A, et al. Relationship Between Pre-transplantation Liver Status and Health-Related Quality of Life After Grafting: A Single-Center Prospective Study; Transplantation Proceedings2014; 46:2770-2773.

\section{Figures}

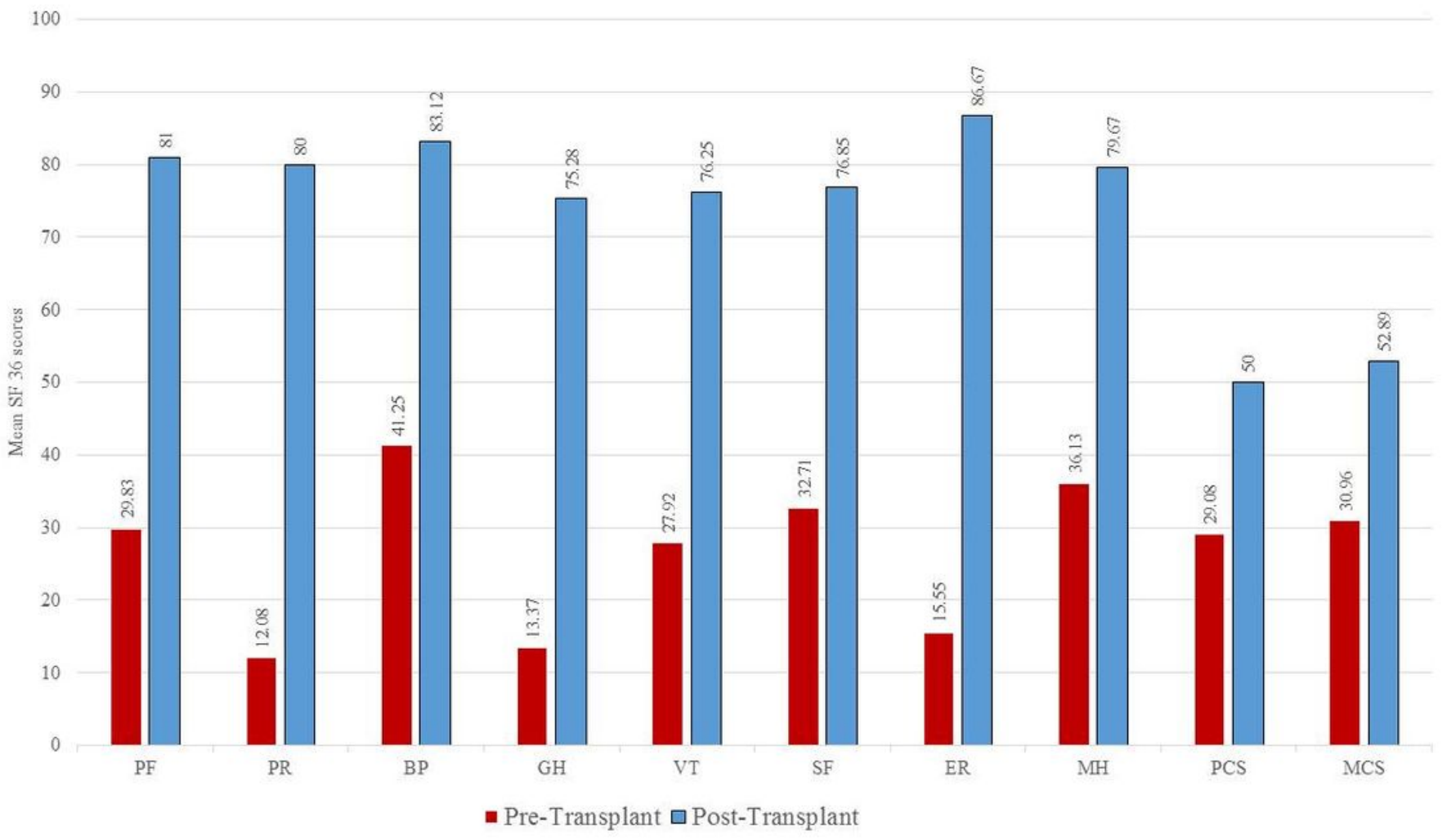

Figure 1

Mean SF36 scores 